\title{
3-(2-Aminophenyl)-4-methyl-1,3-thiazole-2(3H)-thione as an Ecofriendly Sulphur Transfer Agent to Prepare Alkanethiols in High Yield and High Purity
}

\author{
Mohammed Amine Mehdid ${ }^{1}$, Ayada Djafri ${ }^{1}$, Christian Roussel ${ }^{2}$ and Federico Andreoli ${ }^{2, *}$ \\ 1 Laboratoire de synthèse organique appliquée, Département de Chimie, Faculté de Sciences, \\ Université d'Oran-es-Senia, Algeria; E-Mails: mmehdid@yahoo.fr (M.A.M.); \\ djafriayada@yahoo.fr (A.D) \\ 2 ISM2, Chirosciences, Université Paul Cézanne Aix-Marseille III, 13397 Marseille CEDEX 20, \\ France; E-Mail: christian.roussel@univ-cezanne.fr (C.R.)
}

* Author to whom correspondence should be addressed; E-Mail: federico.andreoli@univ-cezanne.fr.

Received: 29 October 2009; in revised form: 10 November 2009 / Accepted: 11 November 2009 / Published: 12 November 2009

\begin{abstract}
A new process is described for preparing very pure linear alkanethiols and linear $\alpha, \omega$-alkanedithiols using a sequential alkylation of the title compound, followed by a ring closure to quantitatively give the corresponding 3-methyl[1,3]thiazolo[3,2-a]$[3,1]$ benzimidazol-9-ium salt and the alkanethiol derivative under mild conditions. The alkanethiol and the heteroaromatic salt are easily separated by a simple extraction process. The intermediate thiazolium quaternary salts resulting from the first reaction step can be isolated in quantitative yields, affording an odourless protected form of the thiols.
\end{abstract}

Keywords: thiols; dithiols; sulphur transfer; thiazoline-2-thione

\section{Introduction}

Long-chain $n$-alkanethiols and $\alpha, \omega$-alkanedithiols are valuable compounds in material sciences due to their ability to bind on gold surfaces [1-8]. In relation with the demand for very pure $n$-alkanethiols and $\alpha, \omega$-alkanedithiols, new methods for the synthesis of these molecules have been developed, furnishing good alternatives to classical ones [9-11]. Among them, the trimethylsilylthioxy- 
dehalogenation reaction reported by $\mathrm{Hu}$ and Fox [12], the direct synthesis from alcohols by Bandgar, Sadavarte and Uppalla [13], and the $\mathrm{SmI}_{2}$-promoted reductions of sodium alkyl thiosulfates and alkyl thiocyanates of Zhan, Lang, Liu and $\mathrm{Hu}$ [14], give alkanethiols in average to good yields; however, for all these methods, distillation or column chromatography is needed to further purify the final product. The one pot conversion of alkyl halides into thiols proposed by Molina, Alajarin and Vilaplana [15] doesn't require final purification steps, but suffers from low yields (lower than 66\%) when applied to the synthesis of long chain $n$-alkanethiols.

Moreover, it must be stressed that thiols easily undergo oxidation to disulphides under ambient conditions. Protecting groups are thus used to grant long term stability to derivatives, implying development of protection-deprotection procedures [16-20] with consequent loss of yield.

Herein we report a new $n$-alkanethiol and linear $\alpha, \omega$-alkanedithiol synthesis which involves the formation of a (bis-)thiazolium salt, starting from 3-(2-aminophenyl)-4-methyl-1,3-thiazole-2(3H)thione (1) and the corresponding (di)halide. The reactions are carried out under mild conditions and no inert conditions are needed; the final products are recovered in their pure form by simple extraction.

\section{Results and Discussion}

3-(2-Aminophenyl)-4-methyl-1,3-thiazole-2(3H)-thione (1) represents a cornerstone for different projects in progress in our laboratory [21-25]. Firstly described by Bellec et al. [26], the synthetic route to 1 starts from readily available material $\left(\mathrm{CS}_{2}\right.$, chloracetone and phenylene-1,2-diamine); this synthesis has been further optimised, leading to crystallised thiazoline-2-thione $\mathbf{1}$ in $75 \%$ isolated yield. We have described that the reaction of 1 with methyliodide gave a salt $2(\mathrm{R}=\mathrm{Me}$, Scheme 1$)$ which was cleanly transformed into thiazolobenzimidazole affording a new and efficient access to that aromatic tricyclic framework [22]. The only by-product was methanethiol, which escaped from the reaction medium and was not characterized. If one considers the two step process described in Scheme 1, the thiazoline-2-thione $\mathbf{1}$ is thus acting as a sulphur transfer agent which mediates the transformation of an alkyl halide into an alkanethiol. The sulphur transfer is occurring through salt 2, which can be considered as a protected form of the alkanethiol. We thus wondered if this procedure might lead to a general and clean synthesis of long-chain $n$-alkanethiols.

A series of different linear alkyliodides was reacted with thiazolinethione $\mathbf{1}$ to produce the derived thiazolium iodides 2 in high yield. In a second step, under heating in refluxing methanol, the thiazolium iodides 2 were quantitatively transformed in a known thiazolobenzimidazolium salt and the corresponding thiols (Scheme 1).

Scheme 1. $n$-Alkanethiols synthesis via thiazolium iodides.

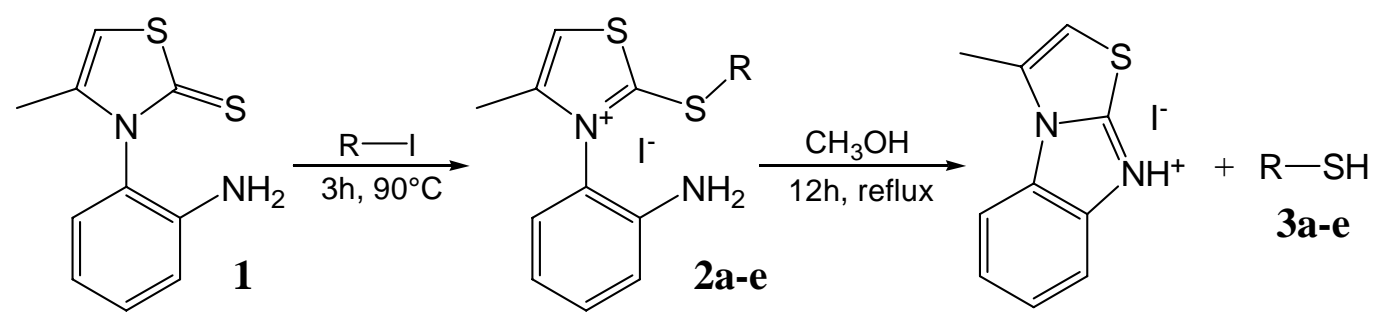


The results obtained are summarized in Table 1.

Table 1. Isolated yields in thiazolium iodides and thiols.

\begin{tabular}{|c|cc|cc|}
\hline Reagent & Thiazolium salt & Yield (\%) & Thiol & Yield (\%) \\
\hline $\mathrm{CH}_{3}\left(\mathrm{CH}_{2}\right)_{6} \mathrm{I}$ & 2a & 96 & 3a & 90 \\
$\mathrm{CH}_{3}\left(\mathrm{CH}_{2}\right)_{8} \mathrm{I}$ & 2b & 96 & 3b & 92 \\
$\mathrm{CH}_{3}\left(\mathrm{CH}_{2}\right)_{9} \mathrm{I}$ & 2c & 94 & 3c & 94 \\
$\mathrm{CH}_{3}\left(\mathrm{CH}_{2}\right)_{11} \mathrm{I}$ & 2d & 93 & 3d & 91 \\
$\mathrm{CH}_{3}\left(\mathrm{CH}_{2}\right)_{17} \mathrm{I}$ & 2e & 98 & 3e & 92 \\
\hline
\end{tabular}

In the first step, the solvent free reaction of thiazoline-2-thione $\mathbf{1}$ with an excess of the corresponding alkyliodide (Scheme 1) generates the respective thiazolium iodides 2. TLC monitoring confirmed that, for all entries, the starting material 1 completely disappeared after $3 \mathrm{~h}$ at $90{ }^{\circ} \mathrm{C}$. Salts 2 were then isolated in very good yields (Table 1) by simple filtration over silica gel and fully characterised. The purification step allows one to recover and to reuse all the excess of alkyliodide, so that the molar ratio $\mathbf{1} /$ (alkylating agent) can be brought closer to $1 / 1$. Moreover, removal of the excess halide from the medium prevents the formation of unwanted dialkylsulphides, once the thiol is formed in the second step. Last but not least, iodides 2 are stable and odourless compounds that can be stored at $3-4{ }^{\circ} \mathrm{C}$ without any risk of alteration, thus the thiazoline moiety acts as a protecting group for the thiol.

Thiazolium iodides 2 are then easily converted by cyclization in methanol under reflux into the thiazolobenzimidazolium iodide in $12 \mathrm{~h}$, releasing the corresponding thiol (second step, Scheme 1). Thiols 3a-e were isolated as pure compounds in yields higher than $90 \%$ (Table 1) by simple extraction with $\mathrm{Et}_{2} \mathrm{O}$. No formation of disulphide by-product was observed in the final products. However when the reaction was performed using various substituted benzylchlorides as alkylating agent for $\mathbf{1}$ a mixture of substituted benzylmercaptans and substituted dibenzyldisulphides was obtained (data not shown).

The good results obtained for $n$-alkanethiols prompted us to apply this method to the synthesis of $\alpha, \omega$-alkanedithiols. In this case, the reaction conditions were changed, due to the need to obtain bisthiazolium salts. Thiazoline-2-thione 1 was then reacted with $1 / 2$ mole of $\alpha, \omega$-alkyldiiodides in chloroform under reflux (Scheme 2).

Scheme 2. Dithiols synthesis via bis-thiazolium diiodides.

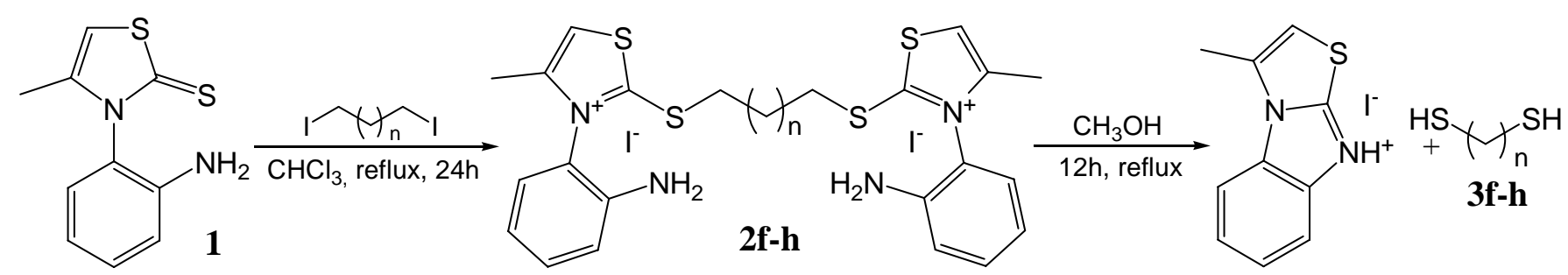


Once again, the complete disappearance of the starting product was monitored by TLC. Two different work ups were successively used to isolate the obtained products (see Experimental section). ${ }^{1}$ H-NMR spectra were recorded for each compound $\mathbf{2 f - h}$, confirming the presence of the corresponding bis-thiazolium diiodides as unique compounds. The salts $\mathbf{2 f - h}$ were then fully characterised and the respective yields are reported in Table 2. It should be noted that bis-thiazolium diiodides 2 might be obtained as a mixture of diastereomers (meso and $d, l$ ) since the starting thiazoline-2-thione $\mathbf{1}$ is chiral [10]. A detailed analysis of this mixture was beyond the scope of this paper since the cyclization step leading to $\alpha, \omega$-alkanedithiols yielded the same achiral compounds starting from either diastereomer.

Table 2. Isolated yields of bis-thiazolium diiodides and $\alpha, \omega$-alkanedithiols.

\begin{tabular}{|c|cc|cc|}
\hline Reagent & Thiazolium salt & Yield (\%) & Thiol & Yield (\%) \\
\hline $\mathrm{I}\left(\mathrm{CH}_{2}\right)_{3} \mathrm{I}$ & $\mathbf{2 f}$ & 94 & $\mathbf{3 f}$ & 91 \\
$\mathrm{I}\left(\mathrm{CH}_{2}\right)_{4} \mathrm{I}$ & $\mathbf{2 g}$ & 95 & $\mathbf{3 g}$ & 90 \\
$\mathrm{I}\left(\mathrm{CH}_{2}\right)_{5} \mathrm{I}$ & $\mathbf{2 h}$ & 89 & 3h & 92 \\
\hline
\end{tabular}

Like thiazolium salts 2a-e, bis-thiazolium salts $\mathbf{2 f - h}$ can be stored at $3-4^{\circ} \mathrm{C}$ without any risk of alteration and show all the advantages mentioned for 2a-e. The same procedure and work up used to obtain thiols is then applied to the synthesis of $\alpha, \omega$-alkanedithiols: after $2 \mathrm{~h}$ under reflux in $\mathrm{MeOH}$, the cyclization to thiazolobenzimidazole is complete and $\alpha, \omega$-alkanedithiols $\mathbf{3 f}$ - $\mathbf{3 h}$ are recovered by simple extraction with $\mathrm{Et}_{2} \mathrm{O}$. It must be stressed that also in this case no formation of by-products derived from sulphur oxidation was observed.

\section{Experimental}

\section{General}

${ }^{1} \mathrm{H}-\mathrm{NMR}$ spectra were recorded at 500,300 or $200 \mathrm{MHz}$ and ${ }^{13} \mathrm{C}-\mathrm{NMR}$ spectra at 125 , 75 or $50 \mathrm{MHz}$ on Bruker Avance DRX-500, DPX-300 or 200 instruments, respectively. Chemical shifts are reported in ppm with the signal for residual solvent as internal standard. $J$ values are reported in $\mathrm{Hz}$. High resolution mass spectra were performed on Q-STAR Elite spectrometer. Melting points were measured using a Büchi Melting Point B-545 apparatus. Filtrations through silica gel were performed with silica gel 60 (230-400 mesh). TLCs were carried out on Merck 60F254 silica plates. 3-(2Aminophenyl)-4-methyl-1,3-thiazole-2(3H)-thione (1) was prepared according to reference [22]. All the halogen compounds were commercially available (Sigma-Aldrich, Alfa Aesar).

\section{General procedure for the synthesis of monothiazolium iodides 2a-e}

3-(2-Aminophenyl)-4-methyl-1,3-thiazole-2(3H)-thione (1, $300 \mathrm{mg}, 1.35 \mathrm{mmol})$ was suspended in the corresponding iodoalkane (for $2 \mathbf{a}$ : $n$-heptyliodide, $2.4 \mathrm{~mL}, 14.64 \mathrm{mmol}$; for $\mathbf{2 b}$ : $n$-nonyliodide, $2.8 \mathrm{~mL}, 14.17 \mathrm{mmol}$; for 2c: $n$-decyliodide, $2.8 \mathrm{~mL}, 13.12 \mathrm{mmol}$; for $\mathbf{2 d}$ : $n$-dodecyliodide, $2.8 \mathrm{~mL}$, $11.35 \mathrm{mmol}$; for $2 \mathrm{e}$ : $n$-octadecyliodide, $3.8 \mathrm{~g}, 9.99 \mathrm{mmol}$ ) and the mixture stirred at $90{ }^{\circ} \mathrm{C}$. After $3 \mathrm{~h}$, the mixture is cooled to r.t. and $\mathrm{CH}_{2} \mathrm{Cl}_{2}(3 \mathrm{~mL})$ is added to completely dissolve the precipitate formed. The solution is filtered on silica gel to remove the excess of alkyl halide (using $\mathrm{CH}_{2} \mathrm{Cl}_{2}$ ). Then the 
silica is washed with $\mathrm{MeOH}$ and the corresponding thiazolium iodide is recovered after evaporation of the solvent.

3-(2-Aminophenyl)-2-(heptylthio)-4-methylthiazol-3-ium iodide (2a): Yield: 96\% (581 mg, orange solid); mp: $53-55^{\circ} \mathrm{C} ;{ }^{1} \mathrm{H}-\mathrm{NMR}\left(300 \mathrm{MHz}, \mathrm{CDCl}_{3}\right) \delta=0.85$ (t, 3H, $J=6.7 ; \mathrm{CH}_{3}$ ), 1.25-1.47 (m, 8H; $\left.4\left(\mathrm{CH}_{2}\right)\right), 1.77-1.88\left(\mathrm{~m}, 2 \mathrm{H} ; \mathrm{CH}_{2}\right), 2.26-2.27\left(\mathrm{~d}, 3 \mathrm{H}, J=1.0 ; \mathrm{CH}_{3}\right), 3.27-3.59\left(\mathrm{~m}, 2 \mathrm{H} ; \mathrm{SCH}_{2}\right), 4.79$ (s, $2 \mathrm{H} ; \mathrm{NH}_{2}$ ), 6.79-6.84 ( m, 1H; Ar), 6.93-7.00 (m, 2H; Ar), 7.33-7.38 (m, 1H; Ar), 7.92 (q, 1H, J=1.0; $=\mathrm{CH}) ;{ }^{13} \mathrm{C}-\mathrm{NMR}\left(75 \mathrm{MHz}, \mathrm{CDCl}_{3}\right) \delta=14.12,14.37,22.59,27.62,28.75,28.83,31.47,36.82,118.37$, 118.53, 118.64, 118.83, 126.87, 133.19, 143.16, 145.67, 177.91; HRMS m/z calcd $\mathrm{C}_{17} \mathrm{H}_{25} \mathrm{~N}_{2} \mathrm{~S}_{2}{ }^{+}$[MI $]^{+}: 321.1454$, found: 321.1455 .

3-(2-Aminophenyl-2-(nonylthio)-4-methylthiazol-3-ium iodide (2b): Yield: 96\% (617 mg, orange solid); mp: $51-53^{\circ} \mathrm{C} ;{ }^{1} \mathrm{H}-\mathrm{NMR}\left(300 \mathrm{MHz}, \mathrm{CDCl}_{3}\right) \delta=0.85\left(\mathrm{t}, 3 \mathrm{H}, J=6.7 ; \mathrm{CH}_{3}\right), 1.23-1.45(\mathrm{~m}, 12 \mathrm{H}$; 6( $\left.\left(\mathrm{CH}_{2}\right)\right), 1.76-1.87\left(\mathrm{~m}, 2 \mathrm{H} ; \mathrm{CH}_{2}\right), 2.26\left(\mathrm{~d}, 3 \mathrm{H}, \mathrm{J}=0.9 ; \mathrm{CH}_{3}\right), 3.26-4.11\left(\mathrm{~m}, 4 \mathrm{H} ; \mathrm{SCH}_{2}+\mathrm{NH}_{2}\right), 6.78-6.84$ ( m, 1H; Ar), 6.93-7.00 ( m, 2H; Ar), 7.32-7.38 ( m, 1H; Ar), 7.93 (q, 1H, $J=0.9 ;=\mathrm{CH})$; ${ }^{13} \mathrm{C}-\mathrm{NMR}(75$ $\left.\mathrm{MHz}, \mathrm{CDCl}_{3}\right) \delta=14.18,14.35,22.71,27.62,28.87,29.09,29.23,29.35,31.87,36.81,118.37,118.52$, $118.65,118.88,126.86,133.17,143.16,145.65,177.88 ;$ HRMS m/z calcd $\mathrm{C}_{19} \mathrm{H}_{29} \mathrm{~N}_{2} \mathrm{~S}_{2}{ }^{+}[\mathrm{M}-\mathrm{I}]^{+}$: 349.1767, found: 349.1767 .

3-(2-Aminophenyl)-2-(decylthio)-4-methylthiazol-3-ium iodide (2c): Yield: 94\% (622 mg, orange solid); mp: $52-54{ }^{\circ} \mathrm{C} ;{ }^{1} \mathrm{H}-\mathrm{NMR}\left(300 \mathrm{MHz}, \mathrm{CDCl}_{3}\right) \delta=0.85$ (t, 3H, $\left.J=6.6 ; \mathrm{CH}_{3}\right), 1.22-1.44$ (m, $14 \mathrm{H}$; $\left.7\left(\mathrm{CH}_{2}\right)\right), 1.76-1.86\left(\mathrm{~m}, 2 \mathrm{H} ; \mathrm{CH}_{2}\right), 2.26\left(\mathrm{~d}, 3 \mathrm{H}, J=0.7 ; \mathrm{CH}_{3}\right), 3.26-3.58\left(\mathrm{~m}, 2 \mathrm{H} ; \mathrm{SCH}_{2}\right), 4.79$ (s, 2H; $\mathrm{NH}_{2}$ ), 6.78-6.83 (m, 1H; Ar), 6.93-7.01 (m, 2H; Ar), 7.32-7.37 (m, 1H; Ar), 7.95 (q, 1H, $J=0.7$; $=\mathrm{CH}) ;{ }^{13} \mathrm{C}-\mathrm{NMR}\left(75 \mathrm{MHz}, \mathrm{CDCl}_{3}\right) \delta=14.17,14.34,22.71,27.60,28.86,29.07,29.31,29.37,29.50$, $31.90,36.79,118.35,118.50,118.63,118.97,129.85,133.14,143.15,145.59,177.81 ; \mathrm{HRMS} \mathrm{m} / \mathrm{z}$ calcd $\mathrm{C}_{20} \mathrm{H}_{31} \mathrm{~N}_{2} \mathrm{~S}_{2}^{+}[\mathrm{M}-\mathrm{I}]^{+}:$363.1923, found: 363.1922 .

3-(2-Aminophenyl)-2-(dodecylthio)-4-methylthiazol-3-ium iodide (2d): Yield: 93\% (651 mg, orange solid); mp: $50-52^{\circ} \mathrm{C} ;{ }^{1} \mathrm{H}-\mathrm{NMR}\left(300 \mathrm{MHz}, \mathrm{CDCl}_{3}\right) \delta=0.86\left(\mathrm{t}, 3 \mathrm{H}, J=6.7 ; \mathrm{CH}_{3}\right), 1.23-1.45$ (m, $18 \mathrm{H}$; $\left.9\left(\mathrm{CH}_{2}\right)\right), 1.77-1.88\left(\mathrm{~m}, 2 \mathrm{H} ; \mathrm{CH}_{2}\right), 2.27\left(\mathrm{~d}, 3 \mathrm{H}, J=0.7 ; \mathrm{CH}_{3}\right), 3.27-3.60\left(\mathrm{~m}, 2 \mathrm{H} ; \mathrm{SCH}_{2}\right), 4.81(\mathrm{~s}, 2 \mathrm{H}$; $\mathrm{NH}_{2}$ ), 6.79-6.84 (m, 1H; Ar), 6.93-6.99 (m, 2H; Ar), 7.33-7.39 ( m, 1H; Ar), 7.90 (q, 1H, $J=0.7$; $=\mathrm{CH}) ;{ }^{13} \mathrm{C}-\mathrm{NMR}\left(75 \mathrm{MHz}, \mathrm{CDCl}_{3}\right) \delta=14.22,14.36,22.77,27.64,28.90,29.11,29.41$ (2C), 29.59, 29.69 (2C), 31.99, 36.84, 118.37, 118.50, 118.64, 118.81, 126.87, 133.18, 143.20, 145.68, 177.96; HRMS m/z calcd $\mathrm{C}_{22} \mathrm{H}_{35} \mathrm{~N}_{2} \mathrm{~S}_{2}^{+}[\mathrm{M}-\mathrm{I}]^{+}: 391.2236$, found: 391.2237 .

3-(2-Aminophenyl-2-(octadecylthio)-4-methylthiazol-3-ium iodide (2e): Yield: 98\% (797 mg, orange solid); mp: $51-53{ }^{\circ} \mathrm{C} ;{ }^{1} \mathrm{H}-\mathrm{NMR}\left(300 \mathrm{MHz}, \mathrm{CDCl}_{3}\right) \delta=0.86\left(\mathrm{t}, 3 \mathrm{H}, J=6.7 ; \mathrm{CH}_{3}\right), 1.18-1.46$ (m, 30H; $\left.15\left(\mathrm{CH}_{2}\right)\right), 1.46-1.78\left(\mathrm{~m}, 2 \mathrm{H} ; \mathrm{CH}_{2}\right), 2.27-2.28\left(\mathrm{~d}, 3 \mathrm{H}, J=0.9 ; \mathrm{CH}_{3}\right), 3.27-3.61\left(\mathrm{~m}, 2 \mathrm{H} ; \mathrm{SCH}_{2}\right), 4.78(\mathrm{~s}$, 2H; $\mathrm{NH}_{2}$ ), 6.79-6.85 (m, 1H; Ar), 6.93-6.99 (m, 2H; Ar), 7.34-7.39 (m, 1H; Ar), 7.88 (q, 1H, $J=0.9$; $=\mathrm{CH}) ;{ }^{13} \mathrm{C}-\mathrm{NMR}\left(75 \mathrm{MHz}, \mathrm{CDCl}_{3}\right) \delta=14.24,14.40,22.80,27.65,28.92,29.13,29.44,29.48,29.62$, 29.73, 29.77 (brs), 29.81 (brs), 32.04, 36.88, 118.36, 118.54, 118.61 (2C), 126.87, 133.23, 143.17, 145.77, 178.05; HRMS m/z calcd $\mathrm{C}_{28} \mathrm{H}_{47} \mathrm{~N}_{2} \mathrm{~S}_{2}^{+}[\mathrm{M}-\mathrm{I}]^{+}: 475.3175$, found: 475.3166 . 
Procedure for the synthesis of bis-thiazolium diiodides $\mathbf{2} \mathbf{f}$ and $\mathbf{2 g}$

3-(2-Aminophenyl)-4-methyl-1,3-thiazole-2(3H)-thione (1, $200 \mathrm{mg}, 89.96 \mathrm{mmol})$ was dissolved in $\mathrm{CHCl}_{3}(5 \mathrm{~mL}$ ), then the corresponding $\alpha, \omega$-diiodide is added (for $2 \mathrm{f}$ : 1,3-diiodopropane, $52 \mu \mathrm{L}, 0.5$ eq; for $\mathbf{2 g}$ : 1,4-diiodobutane, $59 \mu \mathrm{L}, 0.5 \mathrm{eq}$ ) and the solution refluxed under magnetic stirring. After $24 \mathrm{~h}$, the formed precipitate is filtered and washed with $\mathrm{CHCl}_{3}$ to yield the corresponding bis-thiazolium diiodide.

2,2'-(Propane-1,3-diyldisulfanediyl)bis [3-(2-aminophenyl)-4-methyl-1,3-thiazol-3-ium] diiodide (2f): Yield: $94 \%$ (313 mg, pale yellow solid); mp: $178-180^{\circ} \mathrm{C}$ (mixt.); ${ }^{1} \mathrm{H}-\mathrm{NMR}\left(500 \mathrm{MHz}, \mathrm{CD}_{3} \mathrm{OD}\right)$ $\delta=2.25\left(\mathrm{~d}, 6 \mathrm{H}, J=0.9 ; 2 \mathrm{CH}_{3}\right), 2.45-2.52\left(\mathrm{~m}, 2 \mathrm{H} ; \mathrm{CH}_{2}\right), 3.62\left(\mathrm{t}, 4 \mathrm{H}, J=7.2 ; 2\left(\mathrm{SCH}_{2}\right)\right), 6.80-6.83(\mathrm{~m}$, 2H; Ar), 6.99-7.01 (m, 2H; Ar), 7.18-7.21 (m, 2H; Ar), 7.36-7.40 (m, 2H; Ar), 7.86 (q, 2H, J=0.9; $2(=\mathrm{CH})) ;{ }^{13} \mathrm{C}-\mathrm{NMR}\left(125 \mathrm{MHz}, \mathrm{CDCl}_{3}\right) \delta=13.90(2 \mathrm{C}), 27.31,35.00(2 \mathrm{C}), 118.55(2 \mathrm{C}), 118.63(2 \mathrm{C})$, 118.79 (2C), 119.67 (2C), 128.53 (2C), 134.19 (2C), 145.26 (2C), 148.12 (2C), 179.08 (2C); HRMS $\mathrm{m} / \mathrm{z}$ calcd $\mathrm{C}_{23} \mathrm{H}_{26} \mathrm{~N}_{4} \mathrm{~S}_{4}{ }^{2+}[\mathrm{M}-2 \mathrm{I}]^{2+}: 243.0515$, found: 243.0519 .

2,2'-(Butane-1,4-diyldisulfanediyl)bis[3-(2-aminophenyl)-4-methyl-1,3-thiazol-3-ium] diiodide (2g): Yield: 95\% (322 mg, pale yellow solid); mp: 259-261 ${ }^{\circ} \mathrm{C}$ (mixt.); ${ }^{1} \mathrm{H}-\mathrm{NMR}\left(500 \mathrm{MHz}, \mathrm{CD}_{3} \mathrm{OD}\right.$ ) $\delta=2.04-2.09\left(\mathrm{~m}, 4 \mathrm{H} ; 2\left(\mathrm{CH}_{2}\right)\right), 2.25\left(\mathrm{~d}, 6 \mathrm{H}, J=1.1 ; 2 \mathrm{CH}_{3}\right), 3.47-3.54\left(\mathrm{~m}, 4 \mathrm{H} ; 2\left(\mathrm{SCH}_{2}\right)\right), 6.79-6.85(\mathrm{~m}$, 2H; Ar), 6.98-7.02 (m, 2H; Ar), 7.13-7.16 (m, 2H; Ar), 7.35-7.41 (m, 2H; Ar), 7.83 (q, 2H, J=1.1; $2(=\mathrm{CH})$ ); ${ }^{13} \mathrm{C}-\mathrm{NMR}\left(125 \mathrm{MHz}, \mathrm{CDCl}_{3}\right) \delta=13.83(2 \mathrm{C}), 27.74(2 \mathrm{C}), 35.87(2 \mathrm{C}), 118.10(2 \mathrm{C}), 118.71$ (2C), 118.87 (2C), 119.73 (2C), 128.39 (2C), 134.21 (2C), 145.27 (2C), 148.14 (2C), 179.61 (2C); HRMS m/z calcd $\mathrm{C}_{24} \mathrm{H}_{28} \mathrm{~N}_{4} \mathrm{~S}_{4}{ }^{2+}[\mathrm{M}-2 \mathrm{I}]^{2+}: 250.0593$, found: 250.0586 .

Procedure for the synthesis of bis-thiazolium diiodide $\mathbf{2 h}$

3-(2-Aminophenyl)-4-methyl-1,3-thiazole-2(3H)-thione (1, $200 \mathrm{mg}, 89.96 \mathrm{mmol})$ was dissolved in $\mathrm{CHCl}_{3}(5 \mathrm{~mL})$, then 1,5-diiodopentane $(67 \mu \mathrm{L}, 0.5 \mathrm{eq})$ is added and the solution refluxed under magnetic stirring. After $48 \mathrm{~h}$, the solution is cooled to r.t. and $\mathrm{CH}_{2} \mathrm{Cl}_{2}(5 \mathrm{~mL})$ is added. The solution is filtered on silica gel to remove the excess of reactive (using $\mathrm{CH}_{2} \mathrm{Cl}_{2}$ ). Then the silica is washed with $\mathrm{MeOH}$ and bis-thiazolium diiodide $\mathbf{2} \mathbf{h}$ is recovered after evaporation of the solvent.

2,2'-(Pentane-1,5-diyldisulfanediyl)bis[3-(2-aminophenyl)-4-methyl-1,3-thiazol-3-ium] diiodide (2h): Yield: $89 \%$ (308 mg, orange solid); mp: $72-74^{\circ} \mathrm{C}$ (mixt.)C; ${ }^{1} \mathrm{H}-\mathrm{NMR}\left(200 \mathrm{MHz}, \mathrm{CD}_{3} \mathrm{OD}\right) \delta=1.58$ (m, $\left.2 \mathrm{H} ; \mathrm{CH}_{2}\right), 1.89-2.03\left(\mathrm{~m}, 4 \mathrm{H} ; 2\left(\mathrm{CH}_{2}\right)\right), 2.24\left(\mathrm{~d}, 6 \mathrm{H}, J=1.0 ; 2 \mathrm{CH}_{3}\right), 3.46\left(\mathrm{t}, 4 \mathrm{H}, J=7.2 ; 2\left(\mathrm{SCH}_{2}\right)\right)$, 6.77-6.86 (m, 2H; Ar), 6.98-7.03 (m, 2H; Ar), 7.15-7.19 (m, 2H; Ar), 7.34-7.43 (m, 2H; Ar), 7.81 (q, $2 \mathrm{H}, J=1.0 ; 2(=\mathrm{CH})) ;{ }^{13} \mathrm{C}-\mathrm{NMR}\left(50 \mathrm{MHz}, \mathrm{CD}_{3} \mathrm{OD}\right) \delta=13.85(2 \mathrm{C}), 28.20(2 \mathrm{C}), 28.65,36.47(2 \mathrm{C})$, 118.05 (2C), 118.69 (2C), 118.85 (2C), 119.78 (2C), 128.44 (2C), 134.14 (2C), 145.25 (2C), 147.97 (2C), 226.89 (2C); HRMS m/z calcd $\mathrm{C}_{25} \mathrm{H}_{30} \mathrm{~N}_{4} \mathrm{~S}_{4}{ }^{2+}[\mathrm{M}-2 \mathrm{I}]^{2+}: 257.0671$, found: 257.0662.

General procedure for the synthesis of n-alkanethiols

The corresponding thiazolium iodide $(300 \mathrm{mg}$; 2a: $0.67 \mathrm{mmol}$; $2 \mathbf{b}$ : $0.63 \mathrm{mmol}$; 2c: $0.61 \mathrm{mmol}$; 2d: $0.58 \mathrm{mmol}$; $2 \mathrm{e}: 0.50 \mathrm{mmol})$ was dissolved in $\mathrm{MeOH}(2.5 \mathrm{~mL})$ and the solution refluxed under magnetic 
stirring. After $12 \mathrm{~h}, \mathrm{MeOH}$ is evaporated and $\mathrm{Et}_{2} \mathrm{O}(20 \mathrm{~mL})$ is added. The organic layer is washed with $5 \% \mathrm{HCl}(3 \times 15 \mathrm{~mL})$ and brine $(15 \mathrm{~mL})$ and dried over $\mathrm{MgSO}_{4} . \mathrm{Et}_{2} \mathrm{O}$ is then evaporated to yield the corresponding thiols.

1-Heptanethiol (3a) [27]: Yield: 90\% (80 mg, colourless oil); ${ }^{1} \mathrm{H}-\mathrm{NMR}\left(300 \mathrm{MHz}, \mathrm{CDCl}_{3}\right) \delta=0.89$ (t, $\left.3 \mathrm{H}, J=6.7 ; \mathrm{CH}_{3}\right), 1.26-1.43\left(\mathrm{~m}, 9 \mathrm{H} ; 4\left(\mathrm{CH}_{2}\right)+\mathrm{SH}\right), 1.54-1.66\left(\mathrm{~m}, 2 \mathrm{H} ; \mathrm{CH}_{2}\right), 2.49-2.56(\mathrm{~m}, 2 \mathrm{H}$; $\left.\mathrm{SCH}_{2}\right) ;{ }^{13} \mathrm{C}-\mathrm{NMR}\left(75 \mathrm{MHz}, \mathrm{CDCl}_{3}\right) \delta=14.20,22.75,24.81,28.50,28.89,31.88,34.21$.

1-Nonanethiol (3b) [28]: Yield: 92\% (93 mg, colourless oil); ${ }^{1} \mathrm{H}-\mathrm{NMR}\left(300 \mathrm{MHz}, \mathrm{CDCl}_{3}\right) \delta=0.88$ (t, $\left.3 \mathrm{H}, J=6.6 ; \mathrm{CH}_{3}\right), 1.20-1.43\left(\mathrm{~m}, 13 \mathrm{H} ; 6\left(\mathrm{CH}_{2}\right)+\mathrm{SH}\right), 1.55-1.65\left(\mathrm{~m}, 2 \mathrm{H} ; \mathrm{CH}_{2}\right), 2.48-2.55(\mathrm{~m}, 2 \mathrm{H}$; $\left.\mathrm{SCH}_{2}\right) ;{ }^{13} \mathrm{C}-\mathrm{NMR}\left(75 \mathrm{MHz}, \mathrm{CDCl}_{3}\right) \delta=14.24,22.81,24.80,28.53,29.23,29.40,29.62,32.01,34.21$.

1-Decanethiol (3c) [27]: Yield: 94\% (100 mg, colourless oil); ${ }^{1} \mathrm{H}-\mathrm{NMR}\left(300 \mathrm{MHz}, \mathrm{CDCl}_{3}\right) \delta=0.87$ (t, $\left.3 \mathrm{H}, J=6.6 ; \mathrm{CH}_{3}\right), 1.19-1.42\left(\mathrm{~m}, 15 \mathrm{H} ; 7\left(\mathrm{CH}_{2}\right)+\mathrm{SH}\right), 1.55-1.65\left(\mathrm{~m}, 2 \mathrm{H} ; \mathrm{CH}_{2}\right), 2.48-2.55(\mathrm{~m}, 2 \mathrm{H}$; $\left.\mathrm{SCH}_{2}\right) ;{ }^{13} \mathrm{C}-\mathrm{NMR}\left(75 \mathrm{MHz}, \mathrm{CDCl}_{3}\right) \delta=14.23,22.81,24.79,28.53,29.22,29.44,29.66,29.69,32.03$, 34.20 .

1-Dodecanethiol (3d) [27]: Yield: 91\% (106 mg, colourless oil); ${ }^{1} \mathrm{H}-\mathrm{NMR}\left(300 \mathrm{MHz}, \mathrm{CDCl}_{3}\right) \delta=0.88$ $\left(\mathrm{t}, 3 \mathrm{H}, J=6.6 ; \mathrm{CH}_{3}\right), 1.19-1.43\left(\mathrm{~m}, 19 \mathrm{H} ; 9\left(\mathrm{CH}_{2}\right)+\mathrm{SH}\right), 1.55-1.65\left(\mathrm{~m}, 2 \mathrm{H} ; \mathrm{CH}_{2}\right), 2.48-2.56(\mathrm{~m}, 2 \mathrm{H}$; $\left.\mathrm{SCH}_{2}\right) ;{ }^{13} \mathrm{C}-\mathrm{NMR}\left(75 \mathrm{MHz}, \mathrm{CDCl}_{3}\right) \delta=14.26,22.83,24.81,28.54,29.23,29.49,29.67,29.74,29.78$, $29.79,32.06,34.21$.

1-Octadecanethiol (3e) [28]: Yield: 92\% (131 mg, white solid); mp: 30-32 ${ }^{\circ} \mathrm{C} ;{ }^{1} \mathrm{H}-\mathrm{NMR}(300 \mathrm{MHz}$, $\left.\mathrm{CDCl}_{3}\right) \delta=0.88\left(\mathrm{t}, 3 \mathrm{H} J=6.7 ; \mathrm{CH}_{3}\right), 1.19-1.43\left(\mathrm{~m}, 31 \mathrm{H} ; 15\left(\mathrm{CH}_{2}\right)+\mathrm{SH}\right), 1.54-1.67\left(\mathrm{~m}, 2 \mathrm{H} ; \mathrm{CH}_{2}\right)$, 2.47-2.58 (m, 2H; $\left.\mathrm{SCH}_{2}\right) ;{ }^{13} \mathrm{C}-\mathrm{NMR}\left(75 \mathrm{MHz}, \mathrm{CDCl}_{3}\right) \delta=14.27,22.85,24.82,28.54,29.24,29.52$, 29.68, 29.75, 29.82 (3C), 29.85 (5C), 32.08, 34.22.

General procedure for the synthesis of $\alpha, \omega$-alkanedithiols

The corresponding bis-thiazolium diiodide (300 mg; 2f: $0.40 \mathrm{mmol}$; 2 g: $0.40 \mathrm{mmol}$; 2 h: $0.39 \mathrm{mmol}$ ) of are dissolved in $\mathrm{MeOH}(10 \mathrm{~mL})$ and the solution refluxed under magnetic stirring. After $12 \mathrm{~h}$, $\mathrm{MeOH}$ is evaporated and $\mathrm{Et}_{2} \mathrm{O}(20 \mathrm{~mL})$ is added. The organic layer is washed with $5 \% \mathrm{HCl}$ $(3 \times 20 \mathrm{~mL})$ and brine $(15 \mathrm{~mL})$ and dried over $\mathrm{MgSO}_{4} . \mathrm{Et}_{2} \mathrm{O}$ is then evaporated to yield the corresponding dithiols.

1,3-Propanedithiol (3f) [28]: Yield: 91\% (40 mg, colourless oil); ${ }^{1} \mathrm{H}-\mathrm{NMR}\left(300 \mathrm{MHz}, \mathrm{CDCl}_{3}\right) \delta=1.33$ $(\mathrm{t}, 2 \mathrm{H}, J=8.0 ; 2(\mathrm{SH})), 1.86-1.95\left(\mathrm{~m}, 2 \mathrm{H} ; \mathrm{CH}_{2}\right), 2.63-2.70\left(\mathrm{~m}, 4 \mathrm{H} ; 2\left(\mathrm{SCH}_{2}\right)\right) ;{ }^{13} \mathrm{C}-\mathrm{NMR}(75 \mathrm{MHz}$, $\left.\mathrm{CDCl}_{3}\right) \delta=23.01(2 \mathrm{C}), 37.44$.

1,4-Butanedithiol (3g) [27]: Yield: 90\% (44 mg, colourless oil); ${ }^{1} \mathrm{H}-\mathrm{NMR}\left(300 \mathrm{MHz}, \mathrm{CDCl}_{3}\right) \delta=1.35$ $(\mathrm{t}, 2 \mathrm{H}, J=7.8 ; 2(\mathrm{SH})), 1.70-1.76\left(\mathrm{~m}, 4 \mathrm{H} ; 2\left(\mathrm{CH}_{2}\right)\right), 2.51-2.58\left(\mathrm{~m}, 4 \mathrm{H} ; 2\left(\mathrm{SCH}_{2}\right)\right) ;{ }^{13} \mathrm{C}-\mathrm{NMR}(75 \mathrm{MHz}$, $\left.\mathrm{CDCl}_{3}\right) \delta=24.24(2 \mathrm{C}), 32.70(2 \mathrm{C})$. 
1,5-Pentanedithiol (3h) [28]: Yield: 92\% (49 mg, colourless oil); ${ }^{1} \mathrm{H}-\mathrm{NMR}\left(300 \mathrm{MHz}, \mathrm{CDCl}_{3}\right)$ $\delta=1.34(\mathrm{t}, 2 \mathrm{H}, J=7.8 ; 2(\mathrm{SH})), 1.46-1.68\left(\mathrm{~m}, 6 \mathrm{H} ; 3\left(\mathrm{CH}_{2}\right)\right), 2.50-2.57\left(\mathrm{~m}, 4 \mathrm{H} ; 2\left(\mathrm{SCH}_{2}\right)\right) ;{ }^{13} \mathrm{C}-\mathrm{NMR}$ $\left(75 \mathrm{MHz}, \mathrm{CDCl}_{3}\right) \delta=24.60(2 \mathrm{C}), 27.18,33.54(2 \mathrm{C})$.

\section{Conclusions}

We have described a method that offers a new, mild, high yielding and particularly clean way to synthesize $n$-alkanethiols and linear $\alpha, \omega$-alkanedithiols through a sulphur transfer reaction. In addition, the possibility of isolating and storing the intermediate thiazolium salts offers a way to protect thiols in an odourless form.

\section{Acknowledgements}

We thank the "Programme boursier intergouvernemental" B.A.F. (bourse algero-française) for grants to Mohammed Amine Mehdid.

\section{References and Notes}

1. Zamborini, F.P.; Campbell, J.K.; Crooks, R.M. Spectroscopic, voltammetric, and electrochemical scanning tunneling microscopic study of underpotentially deposited $\mathrm{Cu}$ corrosion and passivation with self-assembled organomercaptan monolayers. Langmuir 1998, 14, 640-647.

2. He, H.X.; Zhang, H.; Li, Q.G.; Zhu, T.; Li, S.F.Y.; Liu, Z.F. Fabrication of designed architectures of $\mathrm{Au}$ nanoparticles on solid substrate with printed self-assembled monolayers as templates. Langmuir 2000, 16, 3846-3851.

3. Woehrle, G.H.; Warner, M.G.; Hutchison, J.E. Ligand exchange reactions yield subnanometer, thiol-stabilized gold articles with defined optical transitions. J. Phys. Chem. B 2002, 106, 9979-9981.

4. Shelley, E.J.; Ryan, D.; Johnson, S.R.; Couillard, M.; Fitzmaurice, D.; Nellist, P.D.; Chen, Y.; Palmer, R.E.; Preece, J.A. Dialkyl sulfides: Novel passivating agents for gold nanoparticles. Langmuir 2002, 18, 1791-1795.

5. Snow, A.W.; Ancona, M.G.; Kruppa, W.; Jernigan, G.G.; Foos, E.E.; Park, D. Self-assembly of gold nanoelectronic substrates. J. Mater. Chem. 2002, 12, 1222-1230.

6. Woehrle, G.H.; Hutchison, J.E. Thiol-functionalized undecagold clusters by ligand exchange: Synthesis, mechanism, and properties. Inorg. Chem. 2005, 44, 6149-6158.

7. Rucareanu, S.; Gandubert, V.J.; Lennox, R.B. 4-(N,N-dimethylamino)pyridine-protected Au nanoparticles: Versatile precursors for water- and organic-soluble gold nanoparticles. Chem. Mater. 2006, 18, 4674-4680.

8. Cha, S.-H.; Kim, J.-U.; Kim, K.-H.; Lee, J.-C. Preparation and photoluminescent properties of gold(I)-alkanethiolate complexes having highly ordered supramolecular structures. Chem. Mater. 2007, 19, 6297-6303.

9. Frank, R.L.; Smith, P.V. The preparation of mercaptans from alcohols. J. Am. Chem. Soc. 1946, $68,2103-2104$. 
10. Wardell, J.L. The Chemistry of the Thiol Group; Patai, S., Ed.; Wiley: London, UK, 1974; p. 179.

11. Yamada, M.; Sotoya, K.; Sakakibara, T.; Takamoto, T.; Sudoh, R. Studies on N-alkyl-2(1H)pyridothione. 1. A new synthetic method for thiols. J. Org. Chem. 1977, 42, 2180-2182.

12. $\mathrm{Hu}, \mathrm{J}$.; Fox, M.A. A convenient trimethylsilylthioxy-dehalogenation reaction for the preparation of functionalised thiols. J. Org. Chem. 1999, 64, 4959-4961.

13. Bandgar, B.P.; Sadavarte, V.S.; Uppalla, L.S. Remarkably fast direct synthesis of thiols from alcohols under mild conditions. Chem. Lett. 2000, 1304-1305.

14. Zhan, Z.-P.; Lang, K.; Liu, F.; Hu, L.-M. Water effects on $\mathrm{SmI}_{2}$ reduction: A novel method for the synthesis of alkyl thiols by $\mathrm{SmI}_{2}$-promoted reductions of sodium alkyl thiosulfates and alkyl thiocyanates. Synth. Commun. 2004, 34, 3203-3208.

15. Molina, P.; Alajarin, M.; Vilaplana, M.J. One pot conversion of alkyl halides into thiols under mild conditions. Tetrahedron Lett. 1985, 26, 469-472.

16. Yokoyama, Y.; Takizawa, S.; Nanjo, M.; Mochida, K. Cleavage of a p-cyanobenzyl group from protected alcohols, amines, and thiols using triethylgermyl sodium. Chem. Lett. 2002, 1032-1033.

17. Lin, C.-E.; Richardson, S.K.; Garvey, D.S. L-Cysteine as a water-soluble cation scavenger in the removal of the 2,4,6-trimethoxybenzyl group from thiols. Tetrahedron Lett. 2002, 43, 4531-4533.

18. Behloul, C.; Guijarro, D.; Yus, M. Desilylation procedure via a naphthalene-catalysed lithiation reaction. Tetrahedron 2005, 61, 6908-6915.

19. Behloul, C.; Guijarro, D.; Yus, M. Deallyloxy- and debenzyloxycarbonylation of protected alcohols, amines and thiols via a naphthalene-catalysed lithiation reaction. Tetrahedron 2005, 61, 9319-9324.

20. Holmes, B.T.; Snow, A.W. Aliphatic thioacetate deprotection using catalytic tetrabutylammonium cyanide. Tetrahedron 2005, 61, 12339-12342.

21. Vanthuyne, N.; Andreoli, F.; Fernandez, S.; Roman, M; Roussel, C. Synthesis, chiral separation, barrier to rotation and absolute configuration of $\mathrm{N}$-(o-functionalized-aryl)-4-alkyl-thiazolin-2-one and thiazoline-2-thione atropisomers. Lett. Org. Chem. 2005, 2, 433-443.

22. Roussel, C.; Andreoli, F.; Roman, M; Hristova, M.; Vanthuyne, N. New route to 3alkylthiazolo[3,2-a]benzimidazole derivatives. Molecules 2005, 10, 327-333.

23. Roussel, C.; Roman, M; Andreoli, F.; Del Rio, A.; Faure, R.; Vanthuyne, N. Non-racemic atropisomeric (thio)ureas as neutral enantioselective anion receptors for amino-acid derivatives: Origin of smaller Kass with thiourea than urea derivatives. Chirality 2006, 18, 762-771.

24. Steele, R.M.; Monti, C.; Gennari, C.; Piarulli, U.; Andreoli, F.; Vanthuyne, N.; Roussel, C. Enantioselective cyanosilylation of aldehydes catalysed by a diastereomeric mixture of atropisomeric thioureas. Tetrahedron: Asymmetry 2006, 17, 999-1006.

25. Roussel, C.; Kaid-Slimane, R.; Andreoli, F.; Renaudin, M.; Vanthuyne, N. Synthesis, chiral separation, and absolute configuration of bis-( $N$-aryl $)$ atropisomeric triads: 1,2-bis-[4-methyl-2(thi)oxo-2,3-dihydrothiazol-3-yl]-benzene. Chirality 2009, 21, 160-166.

26. Bellec, N.; Lorcy, D.; Robert, A. Towards functionalised quasi-planar dithiadiazafulvalenes: Synthesis of various precursors. Synthesis 1998, 10, 1442-1446. 
27. National Institute of Advanced Industrial Science and Technology. For comparison of spectroscopic data see: SDBS Web http://riodb01.ibase.aist.go.jp/sdbs/, accessed on 27 October 2009.

28. For comparison of spectroscopic data see: SIGMA-ALDRICH website, http://www.sigmaaldrich.com/, accessed on 13 November 2009.

Sample Availability: Samples of compounds 2a-h and 3a-h are available from the authors.

(C) 2009 by the authors; licensee Molecular Diversity Preservation International, Basel, Switzerland. This article is an open-access article distributed under the terms and conditions of the Creative Commons Attribution license (http://creativecommons.org/licenses/by/3.0/). 